\title{
Radiative falloff in Schwarzschild-de Sitter spacetime
}

\author{
Patrick R. Brady \\ Theoretical Astrophysics 130-33, California Institute of Technology, Pasadena, California 91125 \\ Institute for Theoretical Physics, University of California, Santa Barbara CA 93106 \\ Chris M. Chambers* \\ Department of Physics, Montana State University, Bozeman, Montana 59717 \\ William G. Laarakkers and Eric Poisson \\ Department of Physics, University of Guelph, Guelph, Ontario, Canada N1G 2W1
}

(Draft, January 29, 1999)

\begin{abstract}
We consider the evolution of a scalar field propagating in Schwarzschild-de Sitter spacetime. The field is non-minimally coupled to curvature through a coupling constant $\xi$. The spacetime has two distinct time scales, $t_{e}=r_{e} / c$ and $t_{c}=r_{c} / c$, where $r_{e}$ is the radius of the black-hole horizon, $r_{c}$ the radius of the cosmological horizon, and $c$ the speed of light. When $r_{c} \gg r_{e}$, the field's time evolution can be separated into three epochs. At times $t \ll t_{c}$, the field behaves as if it were in pure Schwarzschild spacetime; the structure of spacetime far from the black hole has no influence on the evolution. In this early epoch, the field's initial outburst is followed by quasi-normal oscillations, and then by an inverse power-law decay. At times $t \lesssim t_{c}$, the power-law behavior gives way to a faster, exponential decay. In this intermediate epoch, the conditions at radii $r \gtrsim r_{e}$ and $r \lesssim r_{c}$ both play an important role. Finally, at times $t \gg t_{c}$, the field behaves as if it were in pure de Sitter spacetime; the structure of spacetime near the black hole no longer influences the evolution in a significant way. In this late epoch, the field's behavior depends on the value of the curvaturecoupling constant $\xi$. If $\xi$ is less than a critical value $\xi_{c}=3 / 16$, the field decays exponentially, with a decay constant that increases with increasing $\xi$. If $\xi>\xi_{c}$, the field oscillates with a frequency that increases with increasing $\xi$; the amplitude of the field still decays exponentially, but the decay constant is independent of $\xi$. We establish these properties using a combination of numerical and analytical methods.
\end{abstract}

Pacs numbers: 04.30.Nk, 04.40.-b, 04.70.Bw

\section{INTRODUCTION AND SUMMARY}

The dynamics of radiative fields in black-hole spacetimes has been investigated since the early 1970's in an effort to understand how asymmetric gravitational collapse proceeds, in the absence of rotation, to form a perfectly spherical black hole. The first work of this type was carried out by de la Cruz, Chase, and Israel [1] , who focused on electromagnetic and linear gravitational perturbations of a spherical collapse. They showed numerically that after an outburst of radiation at the onset of collapse, the radiation decays to zero in a time of the order of the light-crossing time of the resulting event horizon. Subsequently, Price [2] gave an analytical description of the radiative decay, and showed that scalar, electromagnetic, and gravitational radiation all go to zero as an inverse power of time, the power index depending on the multipole order of the perturbation. This late-time decay of the radiation is often referred to as its "power-law tail".

The early investigations were extended and improved by other authors. Bičák [3] generalized Price's analysis and calculated the radiative decay of an electrically neutral scalar field interacting with a charged black hole. He also found an inverse power-law decay of the radiation. Leaver [4] provided a detailed analytical picture of the radiative evolution and introduced much of the mathematical framework adopted in subsequent work. Gundlach, Price, and Pullin [5] provided the most complete picture to date; in addition to describing how the radiation behaves at a fixed distance from the black hole, they also described the behavior at the event horizon and at future null infinity. Furthermore, they numerically simulated the collapse of a self-gravitating scalar field, and showed that the power-law tails are a generic feature of radiative decay, whether a black hole forms or not. These numerical results were reproduced by Burko and Ori [6]. Additional analytical insights can be found in Refs. [7], and various generalizations of the basic scenario have been considered in [10,11.

The works reviewed above were entirely concerned with gravitational collapse to a non-rotating black hole. It is only recently that the analysis was extended to the case of rotating black holes. The first authors to consider radiative decay in Kerr spacetime were Krivan, Laguna, and Papadopoulos 12 (see also [13), who showed numerically that the radiation falls off according to an inverse power law. This inverse power-law behavior was 
confirmed analytically by Hod [14], as well as Barak and Ori [15]

At this point, we have a clear physical picture of how a radiative field behaves during gravitational collapse to a black hole; the picture is essentially the same for scalar, electromagnetic, and gravitational radiation, and it is valid for rotating and non-rotating black holes. There are three stages to the field's dynamical evolution. At the onset of collapse $(t=0)$, an outburst of radiation is emitted; most of this radiation propagates (with distortions) directly to infinity. After this initial outburst, the field oscillates with frequencies and damping times characteristic of the central black hole. This behavior is associated with the field's quasi-normal modes of oscillation, which decay over a time comparable to $100 r_{e} / c$, where $r_{e}$ is the event-horizon radius and $c$ the speed of light. Finally, these oscillations give way to the inverse power-law behavior described above. If the radiative field $\Phi$ is observed at a fixed radius $r$ and the field is static prior to collapse, then $\Phi \sim t^{-(2 l+2)}$ as $t \rightarrow \infty$, where $l$ is the multipole order.

Analytical [4, 7, 9, and numerical [5] studies of radiative dynamics in black-hole spacetimes have revealed that the inverse power-law behavior is not sensitive to the presence of an event horizon. In fact, power-law tails are a weak-curvature phenomenon, and it is the asymptotic structure of the spacetime at radii $r \gg r_{e}$ which dictates how the field behaves at times $t \gg r_{e} / c$. This observation begs the question: how is the field's evolution affected if the conditions at infinity are altered? For example, what happens when the black hole is immersed in an expanding universe 16]?

This question was first addressed by Brady, Chambers, Krivan, and Laguna (hereafter BCKL) in Ref. [17]. They considered the dynamical evolution (both linear and nonlinear) of a scalar field in Reissner-Norsdtröm-de Sitter spacetime, which represents a spherical, charged black hole immersed in de Sitter space - an exponentially expanding universe with a cosmological horizon at a fixed radius $r_{c}$. BCKL found that the scalar field decays not as an inverse power of time, but exponentially at times $t \gg r_{c} / c$. Their numerical results were compatible with the formula $\Phi \sim e^{-l \kappa_{c} t}$, where the decay constant $\kappa_{c} \simeq c / r_{c}$ is the surface gravity of the cosmological horizon. (The surface gravity is precisely defined in Sec. II.) For $l=0$, BCKL found that the field does not decay, but that it settles down to a constant, nonzero value. Therefore, the usual power-law scenario does not necessarily survive when the conditions at infinity are altered.

Our purpose in this paper is to extend the initial work of BCKL; we provide additional details, generalize their discussion, and offer new analytical insights.

We study the dynamics of a scalar field $\Phi$ in Schwarzschild-de Sitter (SdS) spacetime, which describes an electrically neutral black hole (of event-horizon radius $r_{e}$ ) immersed in an exponentially expanding universe (with cosmological-horizon radius $r_{c}$ ). The scalar field satisfies the wave equation

$$
(\square-\xi R) \Phi=0,
$$

where $\square$ is the curved spacetime d'Alembertian operator, $R$ the spacetime's Ricci scalar, and $\xi$ a tunable, nonnegative coupling constant. This equation is more general than the one considered by BCKL, who limited themselves to minimal coupling, i.e., $\xi=0$. We shall see that adding this dimension to the parameter space greatly enhances the number of possible late-time behaviors for the scalar field. Additional details regarding the SdS spacetime and the wave equation (1.1) are presented in Sec. II, which also describes the numerical methods employed to integrate Eq. (1.1).

The analysis presented in this paper establishes the following physical picture. The SdS spacetime comes with two distinct time scales, $r_{e} / c$ and $r_{c} / c$; for the purpose of this discussion we assume that $r_{e} \ll r_{c}$ so that the time scales are cleanly separated. The picture elaborated previously for black holes in asymptotically-flat spacetimes - an initial outburst followed by quasi-normal oscillations followed by power-law tails-continues to hold at times $t \ll r_{c} / c$. In fact, over this time scale, the field propagates as if it were in asymptotically-flat Schwarzschild spacetime. Conditions at radii $r \simeq r_{c}$ have no influence on the field's evolution. At later times, however, when $t$ becomes comparable to $r_{c} / c$, the asymptotic de Sitter structure starts to play a role, and the inverse power-law behavior gives way to a faster, exponential decay. At times $t \gg r_{c} / c$ the conditions at radii $r \gtrsim r_{e}$ no longer have any influence, and the scalar field goes as $\Phi \sim e^{-p \kappa_{c} t}$, where

$$
p=l+\frac{3}{2}-\frac{1}{2} \sqrt{9-48 \xi}+O\left(\frac{r_{e}}{r_{c}}\right) .
$$

Equation (1.2) reduces to $p=l$ when $\xi=0$, in agreement with the BCKL results. In this expression, notice that the square root becomes imaginary when $\xi>\xi_{c}=\frac{3}{16}$. At $\xi=\xi_{c}$, therefore, the scalar field undergoes a transition at which the pure exponential decay observed for $\xi<\xi_{c}$ becomes oscillatory. This surprising property of wave propagation in SdS spacetime was first revealed in our numerical simulations (presented in Sec. III), and then established analytically (as presented in Sec. IV).

Our final section (Sec. V) is devoted to a discussion of the late-time decay of electromagnetic and gravitational radiation in SdS spacetime. If $\Phi$ designates either one of these radiative fields, we argue that $\Phi \sim e^{-p \kappa_{c} t}$ at late times, where $p$ is now given by

$$
p=l+1+O\left(\frac{r_{e}}{r_{c}}\right) .
$$

Thus, both electromagnetic and gravitational radiation behave, at late times, as a conformally invariant $\left(\xi=\frac{1}{6}\right)$ scalar field. 


\section{EQUATIONS AND NUMERICAL METHODS}

The metric of the Schwarzschild-de Sitter (SdS) spacetime is given by

$$
d s^{2}=-f d t^{2}+f^{-1} d r^{2}+r^{2}\left(d \theta^{2}+\sin ^{2} \theta d \phi^{2}\right),
$$

where

$$
f=1-\frac{2 M}{r}-\frac{r^{2}}{a^{2}},
$$

with $M$ denoting the black-hole mass; $a^{2}$ is given in terms of the cosmological constant $\Lambda$ by $a^{2}=3 / \Lambda$. We use geometrized units such that $G=c=1$. The spacetime possesses two horizons: the black-hole horizon is at $r=r_{e}$ and the cosmological horizon is at $r=r_{c}$, where $r_{c}>r_{e}$. The function $f$ has zeroes at $r_{e}, r_{c}$, and $r_{0}=-\left(r_{e}+r_{c}\right)$. In terms of these quantities, $f$ can be expressed as

$$
f=\frac{1}{a^{2} r}\left(r-r_{e}\right)\left(r_{c}-r\right)\left(r-r_{0}\right) .
$$

It is useful to regard $r_{e}$ and $r_{c}$ as the two fundamental parameters of the SdS spacetime, and to express $M$ and $a^{2}$ as functions of these variables. The appropriate relations are

$$
a^{2}=r_{e}^{2}+r_{e} r_{c}+r_{c}^{2}
$$

and

$$
2 M a^{2}=r_{e} r_{c}\left(r_{e}+r_{c}\right) .
$$

We also introduce the surface gravity $\kappa_{i}$ associated with the horizon $r=r_{i}$, as defined by the relation $\kappa_{i}=$ $\frac{1}{2}|d f / d r|_{r=r_{i}}$. Explicitly, we have

$$
\begin{aligned}
& \kappa_{e}=\frac{\left(r_{c}-r_{e}\right)\left(r_{e}-r_{0}\right)}{2 a^{2} r_{e}}, \\
& \kappa_{c}=\frac{\left(r_{c}-r_{e}\right)\left(r_{c}-r_{0}\right)}{2 a^{2} r_{c}}, \\
& \kappa_{0}=\frac{\left(r_{e}-r_{0}\right)\left(r_{c}-r_{0}\right)}{2 a^{2}\left(-r_{0}\right)} .
\end{aligned}
$$

These quantities allow us to write

$$
\frac{1}{f}=\frac{1}{2 \kappa_{e}\left(r-r_{e}\right)}+\frac{1}{2 \kappa_{c}\left(r_{c}-r\right)}+\frac{1}{2 \kappa_{0}\left(r-r_{0}\right)}
$$

and to express the transformation between $r$ and the "tortoise coordinate" $r^{*} \equiv \int f^{-1} d r$ as

$$
\begin{aligned}
r^{*}= & \frac{1}{2 \kappa_{e}} \ln \left(\frac{r}{r_{e}}-1\right)-\frac{1}{2 \kappa_{c}} \ln \left(1-\frac{r}{r_{c}}\right) \\
& +\frac{1}{2 \kappa_{0}} \ln \left(\frac{r}{r_{0}}-1\right) .
\end{aligned}
$$

In terms of $t$ and $r^{*}$ we define the null coordinates $u=$ $t-r^{*}$ (retarded time) and $v=t+r^{*}$ (advanced time) so that the (future) black-hole horizon is located at $u=\infty$, and the (future) cosmological horizon is at $v=\infty$.

We consider a massless scalar field $\Phi$ in the SdS spacetime, obeying the wave equation

$$
(\square-\xi R) \Phi=0,
$$

where $\square=g^{\alpha \beta} \nabla_{\alpha} \nabla_{\beta}$ is the d'Alembertian operator, $R=12 / a^{2}$ the Ricci scalar, and $\xi$ a curvature-coupling constant that we take to be nonnegative. If we decompose the scalar field according to

$$
\Phi=\sum_{l m} \frac{1}{r} \psi_{l}(t, r) Y_{l m}(\theta, \phi),
$$

then each wave function $\psi_{l}$ satisfies the equation

$$
\left(-\frac{\partial^{2}}{\partial t^{2}}+\frac{\partial^{2}}{\partial r^{* 2}}\right) \psi_{l}(t, r)=V_{l}(r) \psi_{l}(t, r),
$$

where the potential function is given by

$$
V_{l}(r)=f\left[\frac{l(l+1)}{r^{2}}+\frac{2 M}{r^{3}}+\frac{2(6 \xi-1)}{a^{2}}\right] .
$$

Using the null coordinates $u$ and $v$, Eq. (2.13) can be recast as

$$
-4 \frac{\partial^{2}}{\partial u \partial v} \psi_{l}(u, v)=V_{l}(r) \psi_{l}(u, v),
$$

in which $r$ is determined by inverting the relation $r^{*}(r)=$ $\frac{1}{2}(v-u)$. Our numerical methods are based on this form for the reduced wave equation.

In order to find a unique solution to Eq. (2.15), initial data must be specified on the two null surfaces $u=0$ (say) and $v=0$ (say). Because the late-time behavior of the wave function is largely independent of the choice of initial data, we set $\psi_{l}(u, v=0)=0$ and use a Gaussian profile, centered on $v_{c}$ and having width $\sigma$, on $u=0$ :

$$
\psi_{l}(u=0, v)=\exp \left[-\frac{\left(v-v_{c}\right)^{2}}{2 \sigma^{2}}\right] .
$$

We numerically integrate Eq. (2.15) using the finitedifferencing scheme suggested by Gundlach, Price, and Pullin [5], in which the coordinates $u$ and $v$ increase by discrete units $\Delta$. In the discrete space, the differential equation becomes

$$
\begin{aligned}
\psi_{l}(N)= & \psi_{l}(W)+\psi_{l}(E)-\psi(S) \\
& -\frac{\Delta^{2}}{8} V_{l}\left(R_{c}\right)\left[\psi_{l}(W)+\psi_{l}(E)\right]+O\left(\Delta^{4}\right),
\end{aligned}
$$

where we have defined the points $N:(u+\Delta, v+\Delta), W$ : $(u+\Delta, v), E:(u, v+\Delta)$, and $S:(u, v)$. The potential is evaluated at the central radius $R_{c}$ corresponding to the off-grid point $\left(u+\frac{1}{2} \Delta, v+\frac{1}{2} \Delta\right)$; thus, $r^{*}\left(R_{c}\right)=\frac{1}{2}(v-u)$.

The computationally expensive part of the calculation resides in the inversion of the relation $r^{*}(r)$, which is 
required to evaluate the potential function $V_{l}(r)$. For values of $r^{*}$ such that $2 \kappa_{e} r^{*}<-1$, we numerically solve the equation

$$
\frac{r}{r_{e}}=1+e^{2 \kappa_{e} r^{*}} \exp \left[\frac{\kappa_{e}}{\kappa_{c}} \ln \left(1-\frac{r}{r_{c}}\right)-\frac{\kappa_{e}}{\kappa_{0}} \ln \left(1-\frac{r}{r_{0}}\right)\right]
$$

by iterations. When $2 \kappa_{c} r^{*}>1$, we use

$$
\frac{r}{r_{c}}=1-e^{-2 \kappa_{c} r^{*}} \exp \left[\frac{\kappa_{c}}{\kappa_{e}} \ln \left(\frac{r}{r_{e}}-1\right)+\frac{\kappa_{c}}{\kappa_{0}} \ln \left(1-\frac{r}{r_{0}}\right)\right]
$$

instead. For all other values of $r^{*}$, we invert the relation $r^{*}(r)$ using the Newton-Raphson method, as implemented in the Numerical Recipes routine rtsafe [18].

Once the integration is completed, the values $\psi_{l}\left(u_{\max }, v\right)$ are extracted, where $u_{\max }$ is the maximum value of $u$ on the numerical grid. If $u_{\max }$ is sufficiently large, then these values give a good approximation for the wave function at the event horizon. Similarly, for $v_{\max }$ sufficiently large, the values $\psi_{l}\left(u, v_{\max }\right)$ give a good approximation for the wave function on the cosmological horizon. Finally, the values of $\psi_{l}$ on the line $u=v-2 k$ are extracted, and expressed as a function of $t=\frac{1}{2}(u+v)=v-k$; here, $k$ is a constant representing the value of $r^{*}$ at which the field is evaluated.

\section{NUMERICAL RESULTS}

\section{A. Behavior at early and intermediate times}

Our first goal in this section is to establish that at times $t \ll r_{c}$, the scalar field behaves as if it were in Schwarzschild spacetime, and that at times $t \sim r_{c}$, a transition occurs in which the inverse power-law decay gives way to an exponential decay. The late times $t \gg r_{c}$ are considered in the next subsection.

Figure 1 displays the behavior of the wave function $\psi_{l}(t, r)$ at a fixed radius $r$, for early and intermediate times. The plots show how the pure Schwarzschild behavior (initial outburst, quasi-normal oscillations, and power-law decay) is distorted by the de Sitter structure of spacetime at large radii. We consider a SdS spacetime with parameters $r_{e}=1$ and $r_{c}=2000$, so that the time scales are well separated. We integrate Eq. 2.15) for $l=0$ and $l=1$, setting $\xi=0$. We compare the field's evolution in SdS spacetime to what it would be in pure Schwarzschild spacetime (also with $r_{e}=1$ ). In both cases the initial data is given by Eq. (2.16), with $v_{c}=10$, $\sigma=3$, and the wave function is evaluated at $r^{*}=10$.

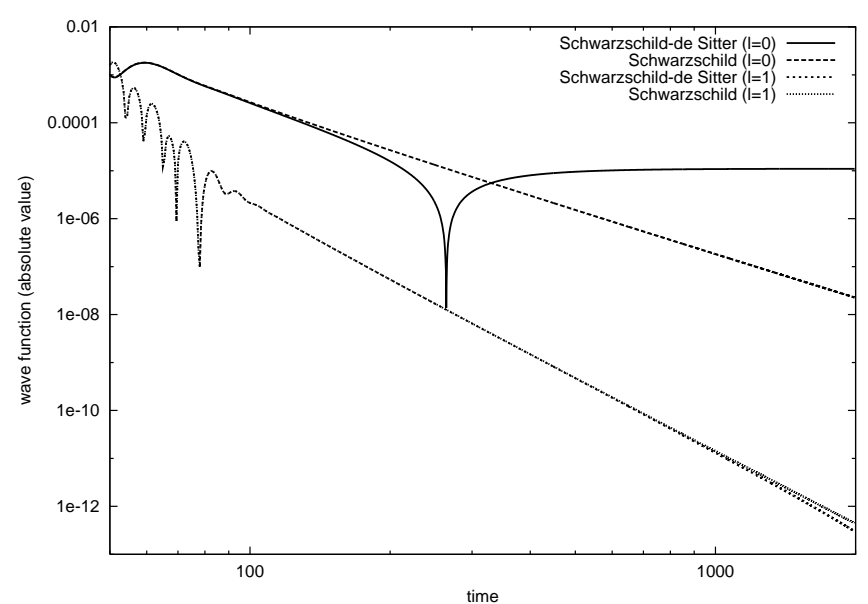

FIG. 1. Absolute value of the wave function $\psi_{l}(t, r)$ as a function of time $t$, evaluated at $r^{*}=10$ in Schwarzschild spacetime $\left(r_{e}=1\right)$ and SdS spacetime $\left(r_{e}=1\right.$ and $\left.r_{c}=2000\right)$. The cases $l=0$ and $l=1$ are considered, and the wave functions are plotted on a log-log scale. In such a plot, a straight line indicates power-law behavior, and a change of sign in the wave function is represented by a deep trough. We see that the early portion of $\psi_{1}$ is oscillatory, and that for SdS spacetime, $\psi_{0}$ changes sign at $t \sim 260$.

The plots show that the physical picture given in Sec. I is accurate. At early times, the Schwarzschild and SdS wave functions display identical behavior, and deviations become apparent only when $t$ becomes comparable to $r_{c}$. For $l=0$, the Schwarzschild behavior $\psi_{0} \sim t^{-3}$ gives way to the wave function changing sign at $t \sim 260$, and settling down to a constant value (equal to $-1.1 \times 10^{-5}$ ) at late times. For $l=1$, the Schwarzschild behavior $\psi_{1} \sim t^{-5}$ is replaced by a faster decay, which eventually becomes exponential. Examination of the field's behavior at the black-hole and cosmological horizons, where $\psi_{l}$ is a function of $u$ and $v$, respectively, reveals precisely the same physical picture.

\section{B. Behavior at late times}

We now consider the behavior of the scalar field at times $t \gg r_{c}$. To ease access to these late times, we consider a SdS spacetime with parameters $r_{e}=1$ and $r_{c}=100$, although this no longer offers a clean separation of the time scales. We begin by integrating Eq. 2.15) with $\xi=0$, for the cases $l=0,1,2$. Once again we set $v_{c}=10, \sigma=3$, and we evaluate the wave function at $r^{*}=10$.

Figure 2 displays the behavior of the wave function $\psi_{l}(t, r)$ at late times. The plots reveal that for $l=0$, the wave function eventually settles down to the constant value $-4.4 \times 10^{-3}$. For $l \neq 0$, the field decays to zero, and our numerical results are compatible with the formula 


$$
\psi_{l}(t) \sim e^{-l \kappa_{c} t}
$$

first discovered by BCKL. The numerically-determined values for the decay constants agree with $l \kappa_{c}$ to within $1 \%$. Multipoles corresponding to $l \geq 3$ could not be examined because the field decays too rapidly and the numerical integration quickly becomes noisy. The latetime behavior of the wave function at the black-hole and cosmological horizons is also described by Eq. (3.1), provided that $t$ is replaced by $v$ on the event horizon, and by $u$ on the cosmological horizon.

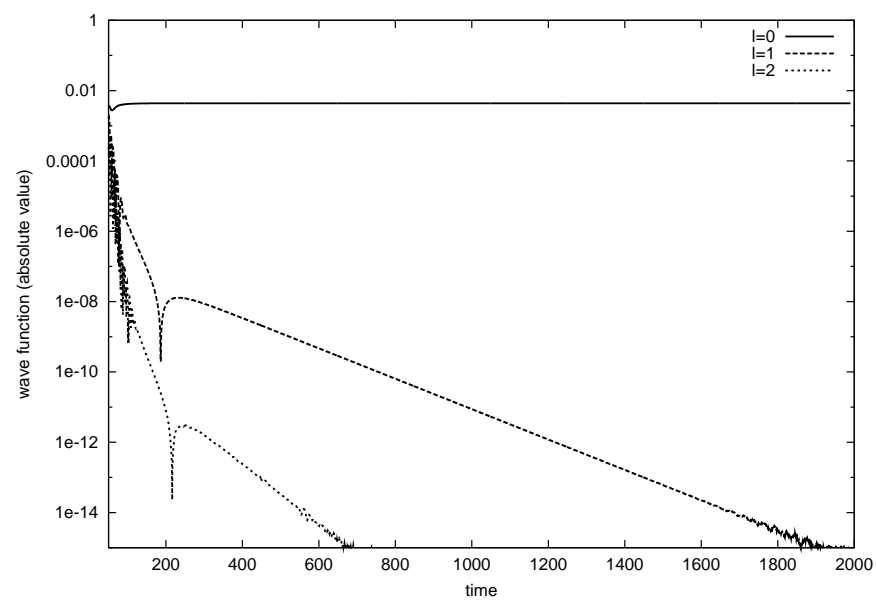

FIG. 2. Absolute value of the wave function $\psi_{l}(t, r)$ as a function of time $t$, evaluated at $r^{*}=10 \mathrm{in} \mathrm{SdS}$ spacetime $\left(r_{e}=1\right.$ and $\left.r_{c}=100\right)$. The cases $l=0,1,2$ are considered, and the wave functions are plotted on a semi-log scale. In such a plot, a straight line indicates exponential behavior. Notice that the final change of sign of the wave function occurs at $t \sim 50$ for $l=0, t \sim 190$ for $l=1$, and $t \sim 220$ for $l=2$. Notice also that the numerical integration becomes noisy when $\left|\psi_{l}\right|$ drops below $10^{-14}$.

A rich spectrum of late-time behaviors is revealed when $\xi$, the curvature-coupling constant, is allowed to be nonzero. This dimension of the parameter space was not explored by BCKL. Figure 3 displays the late-time behavior of $\psi_{0}$ for several values of $\xi$, using the same parameters as before. For $\xi$ smaller than a critical value $\xi_{c}$, the field decays monotonically with a decay constant that increases with increasing $\xi$. When $\xi>\xi_{c}$, however, the wave function oscillates with a decaying amplitude. As $\xi$ is increased away from the critical value $\xi_{c}$, the frequency of the oscillations increases, but the decay constant stays the same. Similar behavior was observed for $l=1$ and $l=2$, and our numerical results are compatible with the formula

$$
\psi \sim e^{-[l+g(\xi)] \kappa_{c} t}
$$

where the function $g(\xi)$ increases with $\xi$ for $\xi<\xi_{c}$, and is complex for $\xi>\xi_{c}$. From our numerical results, we are able to determine that $0.18 \lesssim \xi_{c} \lesssim 0.19$. The analytical calculations presented in Sec. IV show that the function $g(\xi)$ is in fact given by

$$
g(\xi)=\frac{3}{2}-\frac{1}{2} \sqrt{9-48 \xi}+O\left(\frac{r_{e}}{r_{c}}\right)
$$

so that $\xi_{c}=\frac{3}{16}=0.1875$. This relation explains the observed features, and our numerical results agree with Eqs. (3.2) and (3.3) to within 1\%. Equation (3.2), with $t$ replaced by either $v$ or $u$, describes also the behavior of the wave function on the black-hole or cosmological horizons, respectively.

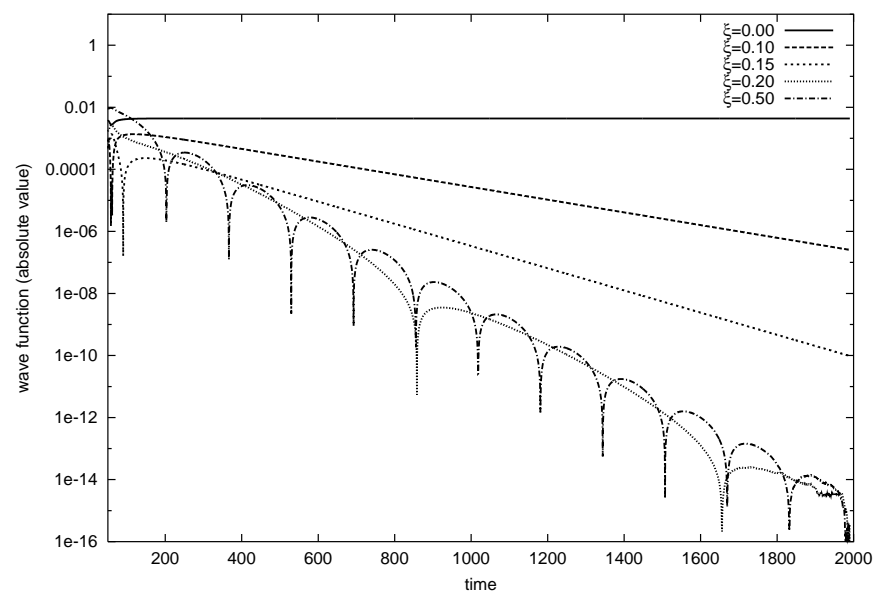

FIG. 3. Absolute value of the wave function $\psi_{0}(t, r)$ as a function of time $t$, evaluated at $r^{*}=10$ in SdS spacetime $\left(r_{e}=1\right.$ and $\left.r_{c}=100\right)$. Several values of $\xi$ are considered, in the interval between $\xi=0$ and $\xi=\frac{1}{2}$. The wave functions are plotted on a semi-log scale. The noteworthy features are these: (i) For $\xi<\xi_{c}$, the wave function decays exponentially, with a decay constant that increases with increasing $\xi$; (ii) for $\xi>\xi_{c}$, the wave function still decays exponentially, but with a decay constant that no longer varies with $\xi$; (iii) for $\xi>\xi_{c}$, the wave function oscillates, with a frequency that increases with $\xi$.

According to the physical picture presented in Sec. I, the field's evolution should proceed as if the field were in pure de Sitter spacetime when $t \gg r_{c}$. In particular, Eqs. (3.2) and (3.3) should describe the late-time behavior of the wave function in de Sitter spacetime. To test this hypothesis, we performed a numerical integration of Eq. (2.15) in pure de Sitter spacetime 19] with a cosmological horizon at $r_{c}=1$, and initial data of the form (2.16) with $v_{c}=1$ and $\sigma=0.1$. The results for $l=0$ are displayed in Figure 4. All the late-time features seen in SdS spacetime are reproduced, and we have verified that the field's evolution is well described by Eqs. (3.2) and (3.3), with $r_{e}=0$ and $\kappa_{c}=1 / r_{c}=1$, to well within $1 \%$. 


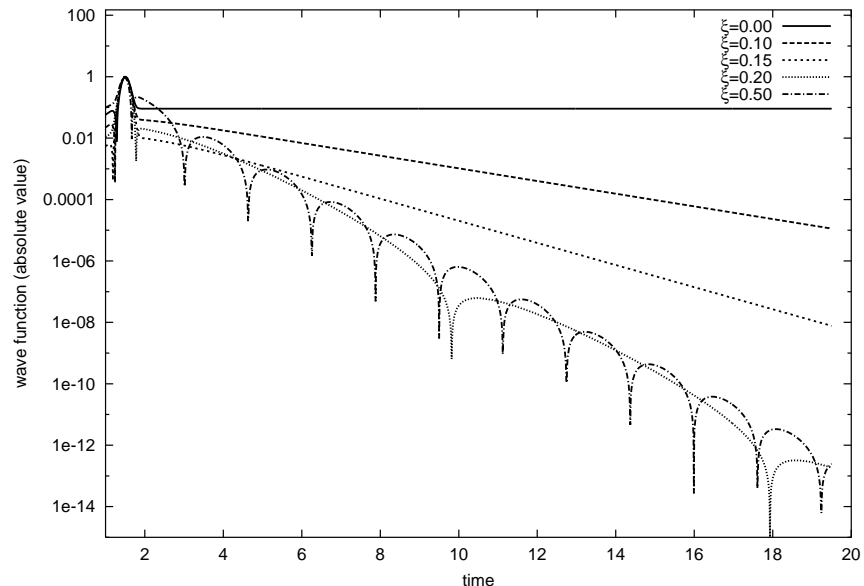

FIG. 4. Absolute value of the wave function $\psi_{0}(t, r)$ as a function of time $t$, evaluated at $r^{*}=0.5$ in pure de Sitter spacetime (with parameter $r_{c}=1$ ). Several values of $\xi$ are considered, in the interval between $\xi=0$ and $\xi=\frac{1}{2}$. The wave functions are plotted on a semi-log scale. These plots show the same features as in the preceding figure.

\section{ANALYTICAL RESULTS}

The observation that the late-time behavior of the scalar field is dictated by the de Sitter structure of spacetime at large radii is the key to the derivation of Eqs. (3.2) and (3.3). Motivated by this observation, we integrate the wave equation (2.13) in pure de Sitter spacetime, and show that the late-time behavior of the wave function is indeed given by Eq. (3.2), with $g(\xi)=\frac{3}{2}-\frac{1}{2} \sqrt{9-48 \xi}$. Apart from an approximation of late times $\left(t \gg r_{c}\right)$, our calculations are exact. That such a treatment is afforded relies on removing the black hole from the spacetime. While this removal produces a large effect at early times, the preceding results indicate that the influence of the central mass is negligible at late times. (We will have to qualify this statement below.) The considerably more difficult job of analytically integrating Eq. (3.2) in full SdS spacetime will be undertaken elsewhere [20].

\section{A. Equations}

We consider a scalar field $\Phi$ in de Sitter spacetime, whose metric is of the form (2.1) with

$$
f=1-r^{2}
$$

where we have set $r_{c}=a=1$ without loss of generality; thus, the surface gravity of the cosmological horizon is $\kappa_{c}=1$. The scalar field obeys the wave equation (2.11), and the reduced wave function $\psi(t, r)$ defined by Eq. (2.12) satisfies Eq. (2.13) with the potential

$$
V(r)=f\left[\frac{l(l+1)}{r^{2}}+2(6 \xi-1)\right] .
$$

We suppress the $l$-label to simplify the notation. In de Sitter spacetime, the relation between $r$ and $r^{*} \equiv$ $\int f^{-1} d r$ is given by

$$
e^{2 r^{*}}=\frac{1+r}{1-r}
$$

We wish to integrate Eq. (2.13) starting with a suitable set of initial conditions at $t=0$. It follows from a simple application of Green's identity that at times $t>0$, the wave function can be expressed as

$$
\psi(t, r)=-\int_{0}^{1}\left[\psi\left(0, r^{\prime}\right) \dot{g}\left(t ; r, r^{\prime}\right)+\dot{\psi}\left(0, r^{\prime}\right) g\left(t ; r, r^{\prime}\right)\right] \frac{d r^{\prime}}{f^{\prime}} .
$$

Here, $\psi\left(0, r^{\prime}\right)$ and $\dot{\psi}\left(0, r^{\prime}\right)$ are the initial data (an overdot indicates time differentiation [21]), and $g\left(t ; r, r^{\prime}\right)$ is the retarded Green's function for the reduced wave equation (2.13); we also use the notation $f^{\prime} \equiv 1-r^{\prime 2}$. The Green's function is zero for $t<0$, and it satisfies Eq. (2.13) with a term $f \delta(t) \delta\left(r-r^{\prime}\right)$ added to the right-hand side.

\section{B. Fourier transform of the Green's function}

Since the late-time behavior of $\psi(t, r)$ is entirely determined by the late-time behavior of $g\left(t ; r, r^{\prime}\right)$, the derivation of Eqs. (3.2) and (3.3) begins with a calculation of the Green's function. We first consider its Fourier transform $\tilde{g}\left(\omega ; r, r^{\prime}\right)$, defined by

$$
g\left(t ; r, r^{\prime}\right)=\frac{1}{2 \pi} \int \tilde{g}\left(\omega ; r, r^{\prime}\right) e^{-i \omega t} d \omega .
$$

For each $\omega$, this function of $r$ and $r^{\prime}$ satisfies the differential equation

$$
\left[\frac{d^{2}}{d r^{* 2}}+\omega^{2}-V(r)\right] \tilde{g}\left(\omega ; r, r^{\prime}\right)=f \delta\left(r-r^{\prime}\right),
$$

where $V(r)$ is given by Eq. (4.2). It is clear that the solution can be expressed in the factorized form

$$
\tilde{g}\left(\omega ; r, r^{\prime}\right)=\frac{1}{W} \psi^{\mathrm{reg}}\left(r_{<}\right) \psi^{\mathrm{up}}\left(r_{>}\right),
$$

where $\psi^{\text {reg }}(r)$ and $\psi^{\text {up }}(r)$ are two linearly independent solutions to the homogeneous form of Eq. (4.6), with $W$ denoting their conserved Wronskian:

$$
W=\psi^{\text {reg }} \frac{d}{d r^{*}} \psi^{\text {up }}-\psi^{\text {up }} \frac{d}{d r^{*}} \psi^{\text {reg }} .
$$

We use the notation $r_{<}=\min \left(r, r^{\prime}\right), r_{>}=\max \left(r, r^{\prime}\right)$, and for simplicity, we do not explicitly indicate the dependence of $\psi^{\text {reg }}, \psi^{\text {up }}$, and $W$ on $\omega$. 
Equation (4.7) correctly represents the retarded Green's function if $\psi^{\mathrm{up}}(r)$ describes waves which are purely outgoing at the cosmological horizon; we choose the normalization

$$
\psi^{\mathrm{up}} \sim e^{i \omega r^{*}} \quad r \rightarrow 1
$$

Also, we must choose for $\psi^{\text {reg }}(r)$ a solution which enforces the boundary condition $\psi^{\text {reg }}(0)=0$; this ensures that the scalar field $\Phi$ is everywhere nonsingular. The calculation of $\tilde{g}\left(\omega ; r, r^{\prime}\right)$ therefore reduces to solving the homogeneous form of Eq. (4.6) for the two functions $\psi^{\text {reg }}(r)$ and $\psi^{\mathrm{up}}(r)$.

\section{Solutions to the homogeneous equation}

The mathematical problem of finding the solutions $\psi^{\text {reg }}(r)$ and $\psi^{\text {up }}(r)$ has been addressed in the literature 22, 23. We shall briefly sketch the method of solution.

The change of variable $z=r^{2}$ and the factorization $\psi=z^{\alpha}(1-z)^{\beta} X$, with $\alpha=\frac{1}{2}(l+1)$ and $\beta= \pm \frac{i}{2} \omega$, transforms the homogeneous form of Eq. (4.6) to a hypergeometric equation for $X$. The fundamental solutions are $X=F\left(a_{ \pm}, b_{ \pm} ; l+\frac{3}{2} ; z\right)$, where $a_{ \pm}=\frac{1}{2}\left(l+g_{+} \pm i \omega\right)$, $b_{ \pm}=\frac{1}{2}\left(l+g_{-} \pm i \omega\right)$, and

$$
g_{ \pm}(\xi)=\frac{3}{2} \pm \frac{1}{2} \sqrt{9-48 \xi}
$$

Both choices of sign in front of $i \omega$ produce a function $\psi(r)$ that goes to zero (as $r^{l+1}$ ) when $r \rightarrow 0$. Choosing the minus sign and the normalization arbitrarily, we set

$$
\psi^{\mathrm{reg}}(r)=r^{l+1}\left(1-r^{2}\right)^{-i \omega / 2} F\left(a_{-}, b_{-} ; l+\frac{3}{2} ; r^{2}\right) .
$$

It is not difficult to show that

$$
\psi^{\mathrm{up}}(r)=2^{i \omega} r^{l+1}\left(1-r^{2}\right)^{-i \omega / 2} F\left(a_{-}, b_{-} ; 1-i \omega ; 1-r^{2}\right)
$$

also satisfies the homogeneous form of Eq. (4.6), and has the asymptotic behavior indicated in Eq. (4.9). On the other hand, the solution

$$
\psi^{\text {down }}(r)=2^{-i \omega} r^{l+1}\left(1-r^{2}\right)^{i \omega / 2} F\left(a_{+}, b_{+} ; 1+i \omega ; 1-r^{2}\right)
$$

satisfies ingoing-wave boundary conditions at the cosmological horizon: $\psi^{\text {down }}(r) \sim e^{-i \omega r^{*}}$ when $r \rightarrow 1$.

These three solutions are not linearly independent. Using Eq. (15.3.6) of Abramowitz and Stegun [24], it is easy to show that

$$
\begin{aligned}
\psi^{\mathrm{reg}}(r)= & \frac{2^{-i \omega} \Gamma\left(l+\frac{3}{2}\right) \Gamma(i \omega)}{\Gamma\left(a_{+}\right) \Gamma\left(b_{+}\right)} \psi^{\mathrm{up}}(r) \\
& +\frac{2^{i \omega} \Gamma\left(l+\frac{3}{2}\right) \Gamma(-i \omega)}{\Gamma\left(a_{-}\right) \Gamma\left(b_{-}\right)} \psi^{\text {down }}(r) .
\end{aligned}
$$

This relation, together with the fact that the Wronskian between $\psi^{\text {down }}(r)$ and $\psi^{\text {up }}(r)$ is equal to $2 i \omega$, imply that

$$
\frac{1}{W}=-\frac{1}{2^{1+i \omega}} \frac{\Gamma\left[\frac{1}{2}\left(l+g_{+}-i \omega\right)\right] \Gamma\left[\frac{1}{2}\left(l+g_{-}-i \omega\right)\right]}{\Gamma\left(l+\frac{3}{2}\right) \Gamma(1-i \omega)} .
$$

\section{Pole structure}

If for the moment we ignore the factor $1 / \Gamma(1-i \omega)$, Eq. (4.15) shows that $1 / W$ [or equivalently $\left.\tilde{g}\left(\omega ; r, r^{\prime}\right)\right]$ is analytic in the complex $\omega$ plane, except for isolated poles in the lower half plane, at

$$
i \omega=i \omega_{n}^{ \pm} \equiv l+g_{ \pm}+2 n,
$$

where $g_{ \pm}(\xi)$ is defined in Eq. (4.10) and $n=0,1,2, \cdots$. There are two poles for each value of the integer $n$. For $\xi<\xi_{c} \equiv \frac{3}{16}, g_{ \pm}$are real functions of $\xi$, and the poles are located along the negative imaginary axis of the complex $\omega$ plane. As $\xi \rightarrow \xi_{c}$, the single poles belonging to the same $n$ merge, becoming a double pole when $\xi=\xi_{c}$. For $\xi>\xi_{c}$, the functions $g_{ \pm}$become complex, and the poles move away from the negative imaginary axis.

The pole nearest to the real axis gives the dominant contribution to the Green's function at late times. For $\xi<\xi_{c}$, this pole is located at $i \omega=l+\frac{3}{2}-\frac{1}{2} \sqrt{9-48 \xi}$. For $\xi=\xi_{c}$, the double pole is located at $i \omega=l+\frac{3}{2}$. For $\xi>$ $\xi_{c}$, there are two nearest poles at $i \omega=l+\frac{3}{2} \pm \frac{i}{2} \sqrt{48 \xi-9}$.

For values of $\xi$ such that $\sqrt{9-48 \xi}$ is an odd integer, the numbers $\omega_{n}^{ \pm}$are also integers. This occurs when $\xi=$ 0 , for which the poles are at $i \omega=l+2 n$ and $i \omega=l+3+2 n$. It also occurs when $\xi=\frac{1}{6}$ (the value of $\xi$ which makes the wave equation conformally invariant), for which the poles are at $i \omega=l+1+2 n$ and $i \omega=l+2+2 n$. These are exceptional cases, because most of these poles are canceled out by the previously ignored factor $1 / \Gamma(1-i \omega)$. This $\Gamma$-function also has integer poles, at $i \omega=1+n^{\prime}$, where $n^{\prime}=0,1,2, \cdots$. It is easy to check that the zeros of $1 / \Gamma(1-i \omega)$ cancel out the poles listed previously, so that $1 / W$ [or equivalently, $\tilde{g}\left(\omega ; r, r^{\prime}\right)$ ] is analytic at these frequencies. The only exception occurs for $l=0$ when $\xi=0$. In this case, the single pole at $i \omega=0$ survives. This is why the scalar field settles down to a nonzero constant at late times.

The pole cancellation which occurs at integer values of $i \omega$ has interesting consequences. For example, consider the case $l=1$, and values of $\xi$ which are small but nonzero. For these values, the pole nearest to the real axis is at $i \omega=1+4 \xi+O\left(\xi^{2}\right)$, and this pole exists as long as $\xi \neq 0$. In the $\xi \rightarrow 0$ limit, however, the wouldbe pole at $i \omega=1$ is canceled by the compensating zero in $1 / \Gamma(1-i \omega)$. We should therefore expect the Green's function - and the scalar field-to display a qualitative change of behavior at late times, as the limit $\xi=0$ is 
taken. Figure 5 shows that such is indeed the case. It is interesting to note that such qualitative changes of behavior do not occur in full SdS spacetime. We return to this point in Sec. IV G.

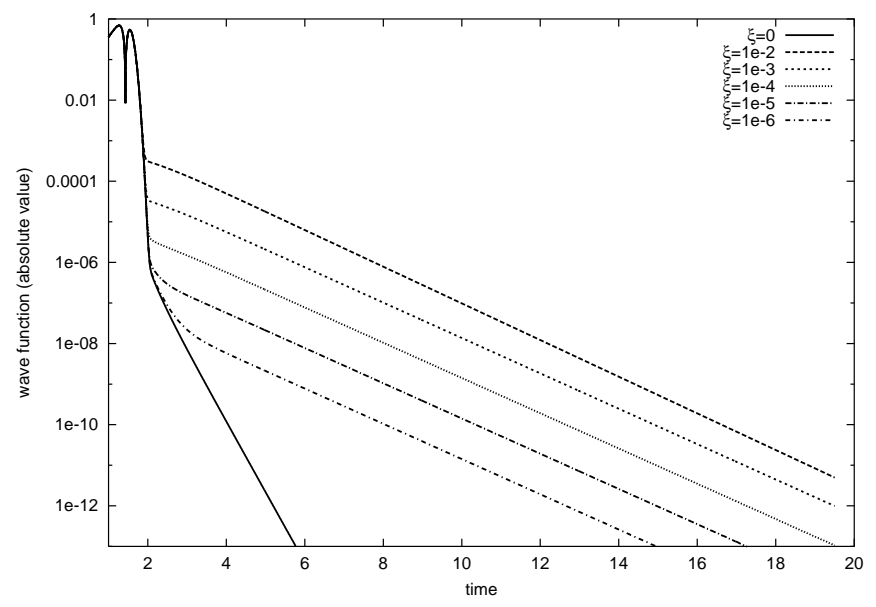

FIG. 5. Absolute value of the wave function $\psi_{1}(t, r)$ as a function of time $t$, evaluated at $r^{*}=0.5$ in pure de Sitter spacetime (with parameter $r_{c}=1$ ). Several small values of $\xi$ are considered, together with the special case $\xi=0$. The plots make it quite clear that the late-time behavior associated with $\xi \ll 1$ is qualitatively different from the behavior associated with $\xi=0$. As explained in the text, this qualitative change of behavior is caused by pole cancellation in $1 / W$.

The presence of poles in $\tilde{g}\left(\omega ; r, r^{\prime}\right)$ makes it very easy to calculate the late-time behavior of the Green's function. Such a calculation is much more difficult when there are no poles, and therefore, this will not be attempted for the special cases $\xi=0$ (except for $l=0$ ) and $\xi=\frac{1}{6}$. In the generic situation (all other cases), the contribution to the Green's function coming from the pole nearest to the real axis is given by

$$
\tilde{g}\left(\omega ; r, r^{\prime}\right) \sim \frac{\Gamma\left(\frac{1}{2} \sqrt{9-48 \xi}\right)}{\Gamma\left(l+\frac{3}{2}\right) \Gamma\left(1-l-g_{-}\right)} \frac{h(r) h\left(r^{\prime}\right)}{i\left(\omega-\omega_{0}\right)},
$$

where

$$
h(r)=r^{l+1}\left(1-r^{2}\right)^{-\left(l+g_{-}\right) / 2}
$$

and

$$
i \omega_{0}=l+\frac{3}{2}-\frac{1}{2} \sqrt{9-48 \xi}
$$

This calculation involves substituting the asymptotic relation $\Gamma(\epsilon) \sim 1 / \epsilon$ in place of the relevant $\Gamma$-function, and evaluating every other factor comprising $\tilde{g}\left(\omega ; r, r^{\prime}\right)$ at the frequency $\omega_{0}$; Eqs. (4.7), (4.11), 4.12), and (4.15) are used along the way 25]. When $\xi=l=0$, Eqs. (4.17)(4.19) reduce to

$$
\tilde{g}\left(\omega ; r, r^{\prime}\right) \sim \frac{r r^{\prime}}{i \omega}
$$

\section{E. Green's function at late times}

The Green's function $g\left(t ; r, r^{\prime}\right)$ can be obtained from $\tilde{g}\left(\omega ; r, r^{\prime}\right)$ by inverting the Fourier transform; this gives rise to Eq. (4.5). The contour of integration is along the real axis of the complex $\omega$ plane. However, because we are interested in the behavior of the Green's function at late times, we can safely close the contour in the lower half plane without changing the result of the integral. The residue theorem then guarantees that $g\left(t, r, r^{\prime}\right)$ can be expressed as a sum over residues, each pole contributing a term proportional to $\exp \left(-i \omega_{n}^{ \pm} t\right)$ to the Green's function. The dominant contribution comes from the pole nearest to the real axis, and we obtain

$$
g\left(t ; r, r^{\prime}\right) \sim-\frac{\Gamma\left(\frac{1}{2} \sqrt{9-48 \xi}\right)}{\Gamma\left(l+\frac{3}{2}\right) \Gamma\left(1-l-g_{-}\right)} h(r) h\left(r^{\prime}\right) e^{-\left(l+g_{-}\right) t},
$$

where $g_{-}(\xi)=\frac{3}{2}-\frac{1}{2} \sqrt{9-48 \xi}$. Near the cosmological horizon, $1-r^{2} \simeq 4 e^{-2 r^{*}}$, and Eq. (4.21) becomes

$$
\begin{aligned}
g\left(t ; r \rightarrow 1, r^{\prime}\right) \sim & -2^{-\left(l+g_{-}\right)} \frac{\Gamma\left(\frac{1}{2} \sqrt{9-48 \xi}\right)}{\Gamma\left(l+\frac{3}{2}\right) \Gamma\left(1-l-g_{-}\right)} \\
& \times h\left(r^{\prime}\right) e^{-\left(l+g_{-}\right) u}
\end{aligned}
$$

where $u=t-r^{*}$. For $\xi=l=0$, both expressions reduce to

$$
g\left(t ; r, r^{\prime}\right) \sim-r r^{\prime}
$$

Equations (4.21)- 4.23) are valid at times $t \gg 1$, or $u \gg$ 1.

We have found that in the generic situation, the latetime behavior of the Green's function is given by $e^{-p \kappa_{c} t}$ or $e^{-p \kappa_{c} u}$, where

$$
p=l+\frac{3}{2}-\frac{1}{2} \sqrt{9-16 \xi} .
$$

Notice that we have re-introduced the scale parameter $\kappa_{c}=1 / r_{c}$. For the special case $\xi=l=0$, the decay constant $p$ is zero, and the Green's function settles down to the time-independent expression given by Eq. (4.23).

Equation (4.24) gives the correct late-time behavior of the Green's function for $\xi<\xi_{c}$ and $\xi>\xi_{c}$. For $\xi=\xi_{c}$, the Green's function is obtained by integrating around a double pole at $i \omega=l+\frac{3}{2}$. This requires integration by parts, and the end result is that for fixed $r<r_{c}$, the Green's function behaves as $\left(\kappa_{c} t\right) \exp \left[-\left(l+\frac{3}{2}\right) \kappa_{c} t\right]$; with $u$ replacing $t$, this is also the correct behavior on the cosmological horizon.

By virtue of Eq. (4.4), we conclude that in pure de Sitter spacetime, the late-time behavior of the wave function $\psi(t, r)$ is also $e^{-p \kappa_{c} t}$ or $e^{-p \kappa_{c} u}$, except for the exceptional cases $-\xi=0$ and $\xi=\frac{1}{6}$ - discussed previously. For the special case $\xi=l=0$, the wave function settles down to a constant value. This conclusion is in full accord with the numerical results presented in Figs. 4 and 5. 


\section{F. The special case $\xi=l=0$}

When $\xi=l=0$, the wave function settles down to a final value $\psi(\infty, r)$ which can readily be calculated using Eqs. (4.4) and (4.23). Re-introducing the scale factor $r_{c}$, we obtain

$$
\psi(\infty, r)=\frac{r}{r_{c}^{2}} \int_{0}^{r_{c}} \dot{\psi}\left(0, r^{\prime}\right) r^{\prime} \frac{d r^{\prime}}{f^{\prime}} .
$$

This equation implies that the value of the scalar field $\Phi$ at late times does not vary with $r$, a necessary consequence of the cosmological no-hair theorems 26 28]. It also implies that $\psi(\infty, r)$ scales as $1 / r_{c}{ }^{2}=\Lambda / 3$, a property that was discussed in BCKL.

Equation (4.25) relates $\psi(\infty, r)$ to the initial data specified on the spacelike surface $t=0$. We can extract more information from this equation, and also test it against our numerical results, if we instead express $\psi(\infty, r)$ in terms of initial data specified on the null surface $u=0$. (We again assume zero data on $v=0$.) To effect this translation, we assume that the initial data has support solely in the "weak-field" region of de Sitter spacetime, in which $\left(r / r_{c}\right)^{2} \ll 1$, so that $f \simeq 1$ and $r^{*} \simeq r$. In this region, and in a neighborhood of the surface $t=0$, we can approximate $\psi(t, r)$ by the flat-spacetime solution $\psi(t, r) \simeq H(v)$, where $H$ is an arbitrary function of $v=t+r$. For this solution, $\dot{\psi}(0, r) \simeq H^{\prime}(r)$ (with a prime indicating differentiation with respect to the argument), and the integral in Eq. (4.25) becomes $\int r d H$. After integration by parts, assuming that $H(v)$ has compact support, we obtain $-\int H(r) d r$, and

$$
\psi(\infty, r) \simeq-\frac{r}{r_{c}^{2}} \int_{0}^{\infty} H(v) d v .
$$

Recall that $H(v)$ is the initial wave profile on the surface $u=0$. For the specific choice (2.16), we obtain

$$
\psi(\infty, r) \simeq-\frac{\sqrt{2 \pi} \sigma}{r_{c}^{2}} r
$$

We have tested this prediction against our numerical results. The accuracy of Eq. (4.27) depends strongly on the value of $v_{c}$, the central position of the Gaussian wave packet. If $r_{c}=1$, we find that for $v_{c}<0.2$, our expression is accurate to within $1 \%$ over a wide selection of values for $r$ and $\sigma$. For $v_{c}>0.2$, the accuracy gets increasingly worse as $v_{c}$ increases; for example, the error grows to $15 \%$ at $v_{c}=0.8$. In view of the fact that Eq. (4.27) was derived under the assumption that the initial data has support only in the region $r \ll r_{c}$ of de Sitter spacetime, this is the expected result.

The rate at which the field $\psi$ settles down to its final constant value was also explored by BCKL. Their numerical results indicated that $\psi-\psi(\infty, r) \sim e^{-q \kappa_{c} t}$ where $q \simeq 2$. Inspection of Eqs. (4.10) and (4.16) reveals that the leading contribution to $\psi-\psi(\infty, r)$ comes from the $n=1$ pole in the Green's function, which produces $q=2+g_{-}=2$. Thus, our analytical results are in full agreement with the BCKL numerical results.

\section{G. Analytical results for SdS spacetime}

Finding the Green's function for a scalar field in SdS spacetime is considerably more difficult than what was accomplished in this section, and this calculation will be the subject of a separate publication [20]. The conclusion is that for SdS spacetime, the late-time behavior of the wave function is still exponential, $\psi \sim e^{-p \kappa_{c} t}$, with the decay constant $p$ given by the same expression as before, apart from corrections of the order of $r_{e} / r_{c}$ :

$$
p=l+\frac{3}{2}-\frac{1}{2} \sqrt{9-48 \xi}+O\left(\frac{r_{e}}{r_{c}}\right) .
$$

This is the result that was quoted in Eqs. (3.2) and (3.3).

In practice, the correction term of order $r_{e} / r_{c}$ is too small to be revealed numerically. Nevertheless, its presence is important, because it prevents $p$ from ever becoming an integer. This means that the cancellation of poles at integer values of $i \omega$, discussed in Sec. IV D, is a phenomenon that occurs only for pure de Sitter spacetime; pole cancellation does not occur in full SdS spacetime. Consequently, the cases $\xi=0$ and $\xi=\frac{1}{6}$ are not exceptional in SdS spacetime, and such qualitative changes of behavior as depicted in Fig. 5 do not occur.

We have numerically tested the validity of Eq. (4.27) for SdS spacetime. As expected, the accuracy of this formula is worse than for pure de Sitter spacetime: With $r_{e}=1, r_{c}=500, \sigma=10$, and evaluating the wave function at $r^{*}=100$, we find that the error is less than $5 \%$ if $100<v_{c}<200$, but that it climbs to approximately $15 \%$ when $v_{c} \sim 50$ or $v_{c} \sim 400$.

\section{ELECTROMAGNETIC AND GRAVITATIONAL RADIATION}

The scalar-wave equation (1.1) serves as a model for the propagation of electromagnetic and gravitational waves in the SdS spacetime. In this section we indicate how our results can be generalized to cover these physically important cases; a more complete discussion will be found in Ref. 20].

It is well known that electromagnetic and gravitational perturbations of the SdS spacetime can be analyzed in terms of a master potential $\Phi$ which is related to the components of the perturbing fields [28]. After a decomposition in spherical harmonics, the wave function $\psi_{l}(t, r)$ is found to obey an equation of the form of Eq. (2.13), but with the generalized potential

$$
V_{l}(r)=f\left[\frac{l(l+1)}{r^{2}}-\frac{2\left(s^{2}-1\right) M}{r^{3}}\right],
$$

where $s=1(s=2)$ for electromagnetic (gravitational) perturbations. In both cases the multipole index $l$ is restricted by $l \geq s$. 
The considerations of the preceding sections indicate that the late-time behavior of $\psi_{l}$ is insensitive to the presence of $M$-dependent terms in Eq. (5.1). Removing these terms, we obtain the potential of Eq. (4.2) with $\xi=\frac{1}{6}$. On these grounds, we expect that the late-time behavior of $\Phi$ is exponential, $\Phi \sim e^{-p \kappa_{c} t}$, with $p$ given by Eq. (1.2) with $\xi=\frac{1}{6}$ :

$$
p=l+1+O\left(\frac{r_{e}}{r_{c}}\right) .
$$

This result is borne out in our numerical simulations.

\section{ACKNOWLEDGMENTS}

P.R.B. was supported by the Sherman Fairchild Foundation, and NSF Grants AST-9417371 and PHY9407194. C.M.C. is grateful to the Royal Commission for the Exhibition of 1851 for financial support. W.G.L and E.P are supported by the Natural Sciences and Engineering Research Council of Canada. E.P. wishes to thank Kip Thorne for his kind hospitality at the California Institute of Technology, where part of this work was carried out.

* Present address: 20 Pritchard Close, Kingsdown, Swindon, United Kingdom SN2 6TZ.

[1] V. de la Cruz, J.E. Chase, and W. Israel, Phys. Rev. Lett. 24, 423 (1970).

[2] R.H. Price, Phys. Rev. D 5, 2419 (1972); 5, 2439 (1972).

[3] J. Bičák, Gen. Relativ. Gravit. 3, 331 (1972).

[4] E.W. Leaver, Phys. Rev. D 34, 384 (1986).

[5] C. Gundlach, R.H. Price, and J. Pullin, Phys. Rev. D 49, 883 (1994); 49, 890 (1994).

[6] L.M. Burko and A. Ori, Phys. Rev. D 56, 7820 (1997).

[7] E.S.C. Ching, P.T. Leung, W.M. Suen, and K. Young, Phys. Rev. Lett. 74, 2414 (1995); Phys. Rev. D 52, 2118 (1995).

[8] N. Andersson, Phys. Rev. D 55, 468 (1997).

[9] L. Barak, Phys. Rev. D 59, 044016 (1999); 044017 (1999).

[10] S. Hod and T. Piran, Phys. Rev. D 58, 024017 (1998); 58024018 (1998); 58024019 (1998).
[11] S. Hod and T. Piran, Phys. Rev. D 58, 044018 (1998).

[12] W. Krivan, P. Laguna, and P. Papadopoulos, Phys. Rev. D 54, 4728 (1996).

[13] W. Krivan, P. Laguna, P. Papadopoulos, and N. Andersson, Phys. Rev. D 56, 3395 (1997).

[14] S. Hod, Phys. Rev. D 58, 104022 (1998); Mode-coupling in rotating gravitational collapse of a scalar field, grqc/9902072 Mode-coupling in realistic rotating gravitational collapse, gr-qc/9902073.

[15] L. Barack and A. Ori, Late-time decay of scalar perturbations outside rotating black holes, gr-qc/9902082.

[16] W.G. Laarakkers and E. Poisson (in preparation).

[17] P.R. Brady, C.M. Chambers, W. Krivan, and P. Laguna, Phys. Rev. D 55, 7538 (1997). Referred to as "BCKL" in the text.

[18] W.H. Press, B.P. Flannery, S.A. Teukolsky, and W.T. Vetterling, Numerical Recipes in $C$ (Cambridge University Press, Cambridge, England, 1986) Chap. 9.

[19] These integrations are much easier to do, owing to the fact that for de Sitter spacetime, the relation $r^{*}=$ $r_{c} \operatorname{arctanh}\left(r / r_{c}\right)$ can be explicitly inverted. On the other hand, special care must be taken with the regular center $r=0$ : The boundary condition $\psi_{l}(t, r=0)=0$ must be imposed, ensuring the regularity of the scalar field $\Phi$ at $r=0$.

[20] E. Poisson (in preparation).

[21] More precisely, the overdot on $g$ denotes differentiation with respect to $t$, while the overdot on $\psi$ denotes differentiation with respect to $t^{\prime}$, which is set to zero in Eq. (4.4). The fact that $g$ depends on $t-t^{\prime}$ explains why the two terms within the square brackets are added instead of subtracted.

[22] D. Polarski, Class. Quantum Grav. 6, 717 (1989); 6, 893 (1989).

[23] D. Lohiya and N. Panchapakesan, J. Phys. A 11, 1963 (1978).

[24] M. Abramowitz and I.A. Stegun, Handbook of Mathematical Functions (Dover, New York, 1970), Chap. 15.

[25] It should be noted that if $\xi>\xi_{c}$, the two poles at $i \omega=$ $l+\frac{3}{2} \pm \frac{i}{2} \sqrt{48 \xi-9}$ are equally near to the real axis, so that both contributions should be taken into account. We leave this subtlety aside, as it does not affect the following discussion.

[26] G.W. Gibbons and S.W. Hawking, Phys. Rev. D 15, 2738 (1977).

[27] S.W. Hawking and I.G. Moss, Phys. Lett. 110B, 35 (1982).

[28] F. Mellor and I. Moss, Phys. Rev. D 41, 403 (1990). 